\title{
DETECTING SUBSIDENCE ALONG A HIGH SPEED RAILWAY BY ULTRASHORT BASELINE TCP-INSAR WITH HIGH RESOLUTION IMAGES
}

\author{
Keren Dai, Guoxiang Liu, Bing Yu,HongguoJia,Deying Ma, Xiaowen Wang
}

Dept. of Remote Sensing and Geospatial Information Engineering, Southwest JiaotongUniversity, Chengdu, Sichuan, 610031, China.-dr.dai_rs@my.swjtu.edu.cn

KEY WORDS: SAR, PS-InSAR, TCP-InSAR, High resolution, Subsidence Detecting, Phase Unwrapping, High Speed Railway

\begin{abstract}
:
A High Speed Railway goes across Wuqing district of Tianjin, China. Historical studies showed that the land subsidence of this area was very serious, which would give rise to huge security risk to the high speed railway. For detecting the detailed subsidence related to the high speed railway, we use the multi-temporal InSAR (MT-InSAR) technique to extract regional scale subsidence of Wuqing district. Take it into consideration that Wuqing district is a suburban region with large area of low coherence farmland, we select the temporarily coherent point InSAR (TCP-InSAR) approach for MT-InSAR analysis. The TCP-InSAR is a potential approach for detecting land subsidence in low coherence areas as it can identify and analysis coherent points between just two images and can acquire a reliable solution without conventional phase unwrapping. This paper extended the TCP-InSAR with use of ultrashort spatial baseline (USB) interferograms. As thetopographic effects are negligible in the USB interferograms, an external digital elevation model (DEM) is no longer needed in interferometric processing, and the parameters needed to be estimated were simplified at the same time. With use of 17 TerraSAR-X (TSX) images acquired from 2009 to 2010 over Wuqing district, the annual subsidence rates along the high speed railway were derived by the USB-TCPInSAR approach. Two subsidence funnels were found at ShuangJie town and around Wuqing Station with subsidence rate of $-17 \sim-27 \mathrm{~mm} / \mathrm{year}$ and $-7 \sim-17 \mathrm{~mm} / \mathrm{year}$, respectively. The subsidence rates derived by USB-TCPInSAR were compared with those derived by the conventional TCP-InSAR that uses an external DEM for differential interferometry. The mean and the standard deviation of the differences between two types of results at 370697 TCPs are $-4.43 \times 10^{-6} \mathrm{~mm} /$ year and $\pm 1.4673 \mathrm{~mm} / \mathrm{year}$, respectively. Further comparison with the subsidence results mentioned in several other studies were made, which shows good consistencies. The results verify that even without using a DEM the USB-TCPInSAR method can detect land subsidence accurately in flat areas.
\end{abstract}

\section{INTRODUCTION}

Multi-temporal interferometric SAR (MT-InSAR) technique is becoming a highly promising approach in detecting land subsidence during the last two decades with an accuracy of millimetre. Several multi-temporal methods and their improvements have been developed and used worldwide, namely PS-InSAR [1],SBAS [2], STUN [3], StaMPS [4] etc. They have been successfully applied to the seismic exploration, tectonic movements, glacier motion, especially to the monitoring of urban surface subsidence that may bring severe hazard.

However, even though some effective improvement have been made by many researchers [5], [6], [7],[8], [9], existing studies have shown that there are two significant limitations of all these methods. One is that these approaches can only be implemented with a number of SAR images covering same area (usually more than 20 images). The phase unwrapping, an essential processing procedure, which cannot be always successfully performed is the other limitation.

Temporarily Coherent Point-InSAR (TCP-InSAR) was proposed in 2011 [10], which can identify coherent points theoretically between just 2 images and can acquire a reliable solution without conventional phase unwrapping. Wuqing district in Tianjin, China was a suburban region with large area of low coherence farmland. As a result, inadequate persistent scatterers usually limit the application of conventional Persistent Scatterers-InSAR (PS-InSAR) on this area. TCPInSAR method can be a good solution to identify more coherent points for calculation and get a reliable results in this area.
This paper used an improved TCP-InSAR with ultrashort baseline (USB-TCPInSAR) approach to detect subsidence along A High Speed Railway. As the topographic effects are negligible in the USB interferograms, an external digital elevation model (DEM) is no longer needed in differential processing, and the parameters need to be estimated was simplified at the same time. With use of 17 TerraSAR-X (TSX) images acquired from 2009 to 2010 over this area, the annual subsidence rates along the railway were derived. Two subsidence funnel that would bring security risks to the railway were found at Shuangjie town and around Wuqing station. These results were remarkably consistent with other studies. The analysis of land subsidence funnel is performed, finding that the over pumping of groundwater caused by industrialization was the main cause. Moreover, in order to confirm the reliability of USB-TCPInSAR, the contrast between USB-InSAR and conventional TCP-InSAR measurements was carried out. The consistent result verified that even without DEM USB-InSAR method can detect land subsidence accurately in flat area.

\section{METHODOLOGY}

\subsection{Selection of Interferometric Pairs with Ultrashort Baselines}

The conventional differential processing generally utilizes an accurate DEM of the imaged surface to remove the topographic fringes from the interferograms (Gabriel et al., 1989; Massonnet \& Feigl, 1998; Rosen et al., 2000; Liu, 2006). However, 
existing DEM cannot provide both satisfactory accuracy and comparable resolution for high resolution images (such as TSX with $2 \mathrm{~m}$ resolution) to remove topographic effects. The height error which cannot be completely compensated by modeling or parameter estimation will propagate into the subsidence measurements thus decrease the accuracies. Therefore, we attempt to choose USB interferometric pairs under certain threshold so as to make the topographic effects negligible to the subsidence measurements.

Assuming that the height error of the DEM used for differential processing is $\Delta h$, the error $\Delta l$ in the resultant LOS motion can be expressed by (Massonnet \& Feigl,1998; Rosen et al., 2000)

$$
\Delta l=\frac{B_{\perp}}{R \cdot \operatorname{Sin} \theta} \cdot \Delta h
$$

Where $B_{\perp}$ denotes the perpendicular baseline length of an interferometric pair, $\theta$ the radar look angle, and $R$ the slant range.

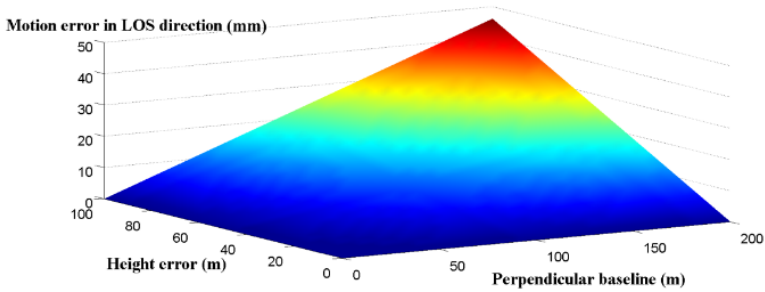

Figure 1. Motion error represented as a function of the perpendicular baseline length and the height error for the case of TSX system

For the case of TSX system with the nominal imaging parameters, i.e., $\theta=41^{\circ}, R=662520 \mathrm{~m}$, Figure 1 shows the functional relationship of $\Delta l, \Delta h, B_{\perp}$. It can be observed that $\Delta l$ is not sensitive to $\Delta h$ while $B_{\perp}$ is very short. $\Delta l$ only varies between 0 and $3 \mathrm{~mm}$ if $B_{\perp}$ is less than $15 \mathrm{~m}$ even $\Delta h$ is $100 \mathrm{~m}$. It is therefore can be concluded that a TSX interferometric pair with $B_{\perp}$ less than $15 \mathrm{~m}$ can be used to generate a USB interferogram that is less sensitive to topographic effects[11].

\subsection{TCP Identification and Networking}

The TCPs are defined as the ground targets that appear in all the interferograms or only some of the interferograms. Amplitudes or other deviation indexes proposed by Ferretti et al. (2001) are no longer used as criteria in TCP selection.

Offset, an important parameter in the process of co-registration between two SAR images, was innovatively introduced as a criteria for TCP identification. Existing studies have shown that in the amplitude correlation method the estimation of the local image offsets is reliable only when the features in the two SAR image patches are identical (Strozzi et al., 2002) and the estimated offsets of points with high coherence are less sensitive to the size of the patches and to the oversampling factors (Zhang et al., 2011).

Taking advantage of these two properties, therefore,we can estimate the offsets by changing the size of the image patches in cross-correlation estimation and then calculate the standard deviation of an estimated offset. At last offsets are fitted to a smooth polynomial. TCPs are selected after these three procedures.
Once all the TCPs are identified, a network was formed by connecting the adjacent TCPs (each connection is regarded as an arc). It should be noted that the length of arcs should be limited in TCP solution. Local Delaunay Triangulation network is the optimum network in consideration of the computation burden and the reliability for TCP solution. For more details about TCP identification and networking, you can consult Zhang et al. (2011b).

\subsection{USB-TCPInSAR simplified Modeling and Least Squares Solution}

Let us assume that the land subsidence is of linear accumulation in time and there is no DEM error due to the low sensitivity of topography in USB interferograms. The differential interferometric phase at an arbitrary pixel with coordinates $(x, y)$ for the $i$ th interferogram can be modelled as,

$$
\phi_{x, y}^{i}=W\left\{\phi_{\text {defo, } x, y}^{i}+\phi_{\text {res }}^{i}\right\}
$$

Where $\mathrm{W}\{\}$ denotes the wrapping operator. Compared with the conventional TCP-InSAR model proposed by LeiZhang, which modelled the differential interferometric phase as $\phi_{x, y}^{i}=W\left\{\phi_{\text {topo }, x, y}^{i}+\phi_{\text {defo, } x, y}^{i}+\phi_{\text {res }}^{i}\right\}$ [12], (2) ignored the parameter $\phi_{\mathrm{topo}, x, y}^{i}$, simplifying the model and the consequent solution.

The line-of-sight(LOS) displacement of $\mathrm{TCP}(\mathrm{x}, \mathrm{y})$ in each interferogram can be described by a linear combination of the mean deformation rate between the arbitrary master (M) and slave(S) images and the corresponding time span, i.e. $v\left(t_{m}, t_{s}\right)=V$.Thus, the corresponding phase for the displacement is

$$
\phi_{d e f o, x, y}^{i}=-\frac{4 \pi}{\lambda}\left(t_{m}-t_{s}\right) V=\beta^{i} V
$$

Moreover, the residual phase $\phi_{\text {res }}^{i}$ can be viewed as the sum of several components, including atmospheric delay $\phi_{a t m o, x, y}^{i}$, azimuth Doppler centroid difference $\phi_{d o p, x, y}^{i}$,orbit errors $\phi_{\text {orbit } x, y, y}^{i}$, and decorrelation noise $\phi_{n o i s e, x, y}^{i}$. Therefore (2) can be expressed as

$$
\phi_{x, y}^{i}=-\frac{4 \pi}{\lambda} \sum_{k=1}^{C_{i}-1}\left(t_{k}-t_{k-1}\right) v_{k}+\phi_{\text {atmo }, x, y}^{i}+\phi_{o r b i t, x, y}^{i}+\phi_{n o i s e, x, y}^{i}+\phi_{d o p, x, y}^{i}
$$

Since we construct a local Delaunay triangulation network to connect pairs of TCPs, the phase difference of an arc is given by,

$$
\Delta \phi_{(x, y),\left(x^{\prime}, y^{\prime}\right)}^{i}=\beta^{i} \Delta V_{(x, y),\left(x^{\prime}, y^{\prime}\right)}^{i}+\Delta \phi_{r e s,(x, y),\left(x^{\prime}, y^{\prime}\right)}^{i}
$$

where

$\Delta \phi_{r e s,(x, y),\left(x^{\prime}, y^{\prime}\right)}^{i}=\Delta \phi_{\text {atm, }(x, y),\left(x^{\prime}, y^{\prime}\right)}^{i}+\Delta \phi_{o r b i t,(x, y),\left(x^{\prime} y^{\prime}\right)}^{i}+\Delta \phi_{\text {noise, }(x, y),\left(x^{\prime}, y^{\prime}\right)}^{i}+\Delta \phi_{d o p,(x, y),\left(x^{\prime}, y^{\prime}\right)}^{i}$ Since the atmospheric artifacts are strongly correlated in space, the differential atmospheric contributions between a pair of nearby TCPs are expected to be small [Li et al., 2006; Williams et al., 1998]. The differential orbital component generally have a similar characteristic. Moreover, the differencing operation can significantly reduce the effect of Doppler centroid difference. Therefore, $\Delta \phi_{r e s,(x, y),\left(x^{\prime}, y^{\prime}\right)}^{i}$ can be safely taken as a random variable with an expectation $\mathrm{E}\left(\Delta \phi_{r e s,(x, y),\left(x^{\prime}, y^{\prime}\right)}^{i}\right)=0$ (Zhang et al., 2011).Therefore, the system of observation equations can be written as

$$
\Delta \phi=\beta \cdot \Delta V+\phi_{\text {res }}
$$

$\Delta V$ can be solved by a least square (LS) approach, which can be expressed as

$$
\Delta V_{(\mathrm{x}, \mathrm{y})}=\left(\mathrm{A}^{T} \mathrm{P}^{d d} \mathrm{~A}\right)^{-1} A^{T} P^{d d} \Delta \phi
$$

Where $\mathrm{P}^{d d}$ is the priori weight matrix which can be obtained by 
taking the inverse of the variance matrix $Q^{d d}$ of the observations. The $Q^{d d}$ can be derived from the standard deviations in offsets estimated during the TCP identification. For more detailed about solution, you can consult [12].

\section{STUDY AREA AND DATA SOURSE}

Tianjin is one of four municipalities which located in northeast of China near to the Bohai Bay. It suffers water shortage due to its natural geographic condition and semi-arid climate (EnviroLibrary, 2008). To meet the industrial and agricultural needs, a large amount of groundwater has been over pumped since 1920s, thus leading to severe land subsidence in many areas of Tianjin (Enviro-Library, 2008).

A High Speed Railway is the start of Chinese high-speed railway era, with the world's most advanced non-ballasted track technology. The subsidence along the high speed railway would cause subsidence damage and give rise to huge security risk especially to this $350 \mathrm{~km} / \mathrm{h}$ high speed railway. Detecting the subsidence of railway, therefore, is a very important task. We select Wuqing District located in the northwest of Tianjin as study area because of the extensive groundwater exploitation and historical land subsidence occurring in this region,.

To analyse subsidence along railway of this areausing USB TCP-InSAR method, we selected 17 TSX SAR images with a resolution of about $2 \mathrm{~m}$ in stripmap mode between April 2009 and November 2010. As shown in Figure 2, the study area is framed by a red square (about $22 \times 22 \mathrm{~km}^{2}$ ) in the northwest part of Tianjin, while A High Speed Railway is highlighted by a blue line.

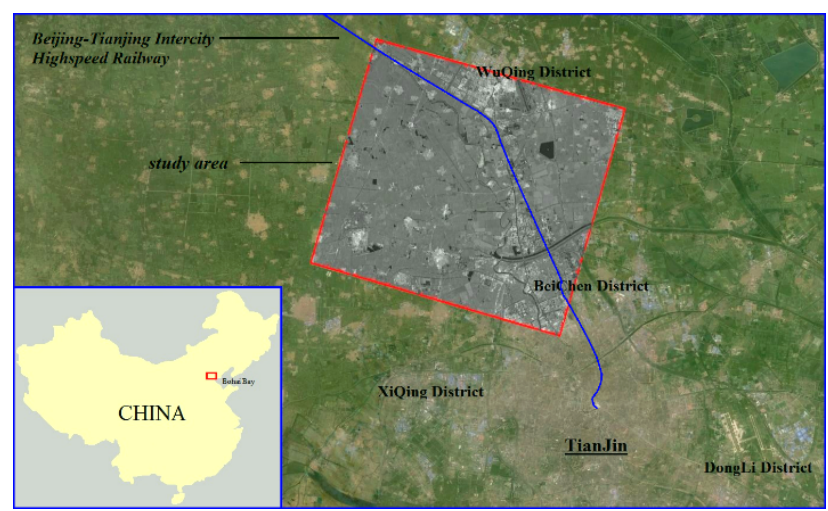

Figure 2.Location map of the study area

\section{RESULT AND DISCUSSION}

\subsection{Derive Subsidence Rates Map by USB-TCPInSAR}

17 USB interferometric pairs with spatial baselines of less than $15 \mathrm{~m}$ were selected as shown in Figure 3. The colour bar indicates the variation of spatial baseline and each connected horizontal line indicates the temporal baseline for the corresponding interferometric pair. It can be seen that the temporal baselines range from 43 days to 207 days.

By following the procedures as described in chapter 2, 1201586 TCPs were identified and formed a Local Delaunay Triangulation network. It should be noted that as the USB pairs are not sensitive to topographic effects including the high-rise buildings, thus an external DEM is not used in the interferometry process or any other subsequent processes. The LS approach was performed with the improved Least Squares Solution model that only had deformation rate parameterized. The resultant subsidence rateat all the valid TCPs are shown in Figure 4.

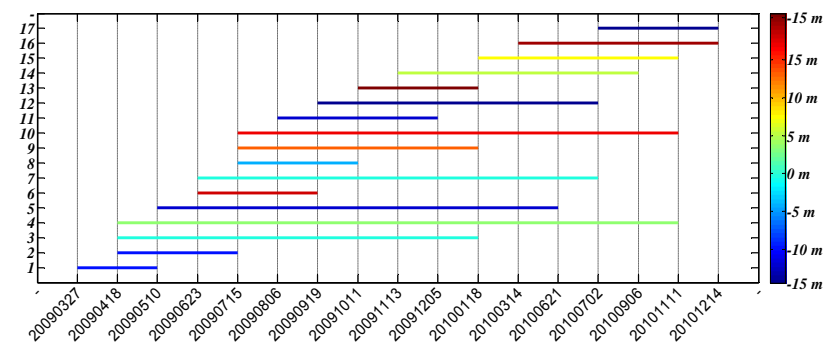

Figure 3. The spatial and temporal baselines of the 17 USB interferometric pairs used for TCP solution

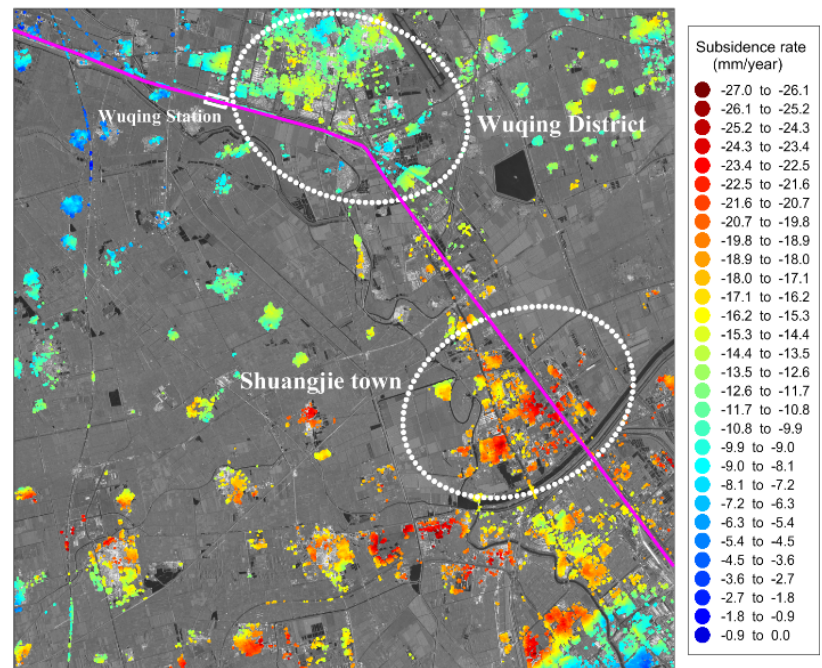

Figure 4. Subsidence rates estimated for all the valid TCPs

A High Speed Railway is highlighted by a pink line and the Wuqing Station is located in the southwest of Wuqing District marked by a white hollowrectangle. Two subsidence funnel circled along the railway line can been found.

\subsection{Subsidence Analysis}

The process of land subsidence is a complex phenomenon which has multiple causes. Existing studieshave showed that in Tianjin and its surroundings, overpumping of groundwater is the main cause leading to land subsidence. Data of the annual groundwater pump rate and of theannual subsidence rate were collected to discuss the relationship between ground water pumping and land subsidence in Tianjin as shown in Figure 5.

It is clearly seen that these two numerical values correlate very well, indicating that the dominant cause of land subsidence in Tianjin is groundwater pumping. The amount of groundwater pumped, therefore, will be given the first consideration when analysing the land subsidence in Tianjin and its surrounding area.

The northern subsidence funnel is in the Wuqing District where Wuqing station located. Wuqing District was one of the most groundwater over pumped area looking through the history. The recent ( 2007) average subsidence rate was 35-45 mm/year and the maximum cumulative subsidence was $2.1 \mathrm{~m}$ from 1959 to 
2007 [15]. A systematic program of prevention and control was carried out since the 1970 s by the Tianjin and the central government, such as importing substitute surface water, artificial recharge of aquifers. From our result we can observe that the subsidence in Wuqing District is around -7 -17 $\mathrm{mm} / \mathrm{year}$, which decrease dramatically compared to history own to relevant policies.

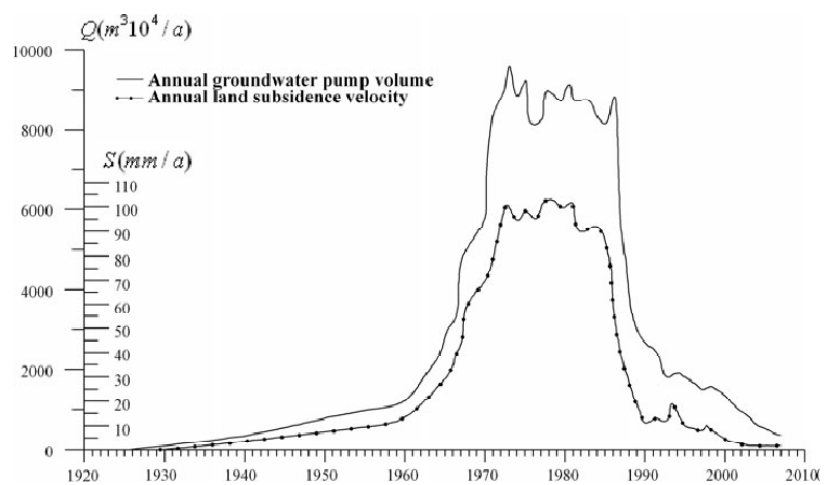

Figure 5. The relation curve of annual land subsidence rate and annual groundwater pump volume in Tianjin (Source: Tianjin Land Subsidence Control Office 1989-2005)

The other subsidence funnel is in the Shuangjie town with the subsidence of -17 -27 mm/year. Shuangjie town was an industrial zone where the groundwater pumping yield has drastically increased as a result of rapid economic development and industrial modernization. Until now, Shuangjie town industrial area was built with five industrial district, with a total area of 3300 acres (approximately 220.1 hectares). National Beichen Science and Technology Park is located in the region of the town. Shuangjie town plan to gradually build industrial cluster with pillar industry of mechanical and electrical, metallurgical processing, equipment manufacturing etc. Several large transnational enterprises have invested and set up plants in these area, such as Dingjin Group, Robust, MetalKorea.

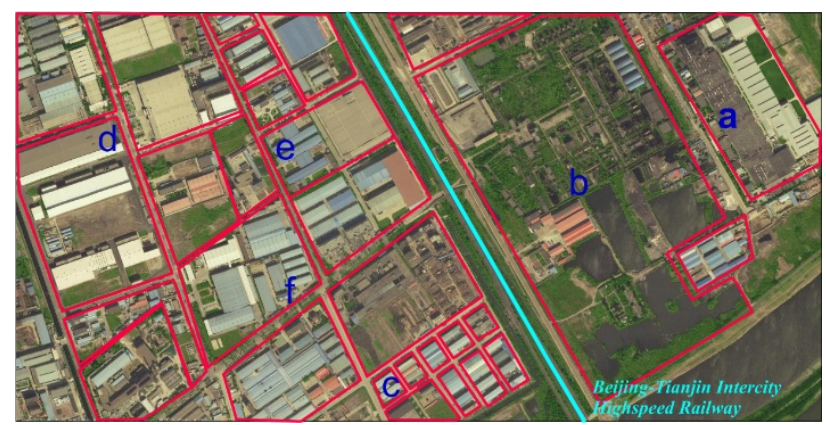

Figure 6. Industrialization of Shuangjie town

Table 1. Some companies in the town

\begin{tabular}{|l|l|}
\hline a & Bridgestone Corporation \\
\hline b & Tianjin Dolphin Rubber Company \\
\hline c & Alcatel factory \\
\hline d & Siemens factory \\
\hline e & Robust Corporation \\
\hline f & Tianjin Ruida Wire \& Cable Works \\
\hline
\end{tabular}

Figure 6 shows a typical features of Shuangjie town and we can see a large numbers of industrial factory buildings appearing in the town. A High speed Railway was marked by a blue line and each red polygon marked an industrial park or factory. The Table 1 below listed some domestic and international companies according to our investigation.
Consequently, Shuangjie town has experienced extensive groundwater withdrawal for water resources due to the industrialization, and this has indeed induced disasters like land subsidence damage. The detailed subsidence map show fairly consistent patterns with other studies of other methods, like [13] [14]. In the TCPInSAR method, more TCPs have been identified and the high resolution result can help government make compensation strategies better and guild engineering construction.

\subsection{Contrast and Discussion}

In order to confirm the reliability of the improved USB TCPInSAR without DEM, we use conventional TCP-InSAR proposed by Zhanget al. (2011b) to do the calculation with same interferometric pairs. 370697 common TCPs were randomly chosen and the histogram indicating the differences of subsidence rates at these TCPs were shown in Figure 7. The value of the differences range from -10.0727 to $6.9530 \mathrm{~mm} / \mathrm{year}$. It should be noted that Figure 7 only shows the points with difference in the interval $(-5,5) \mathrm{mm} / \mathrm{year}$, which already contains $368396(99.38 \%)$ points.

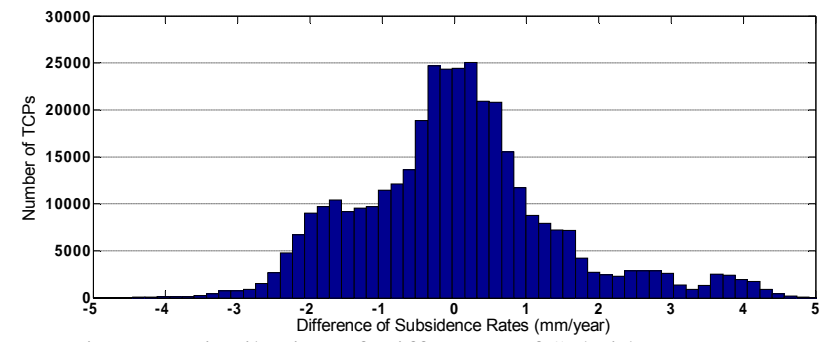

Figure 7.Distribution of Difference of Subsidence Rates

The mean and the standard deviation of the differences are -4.43 $\times 10^{-6} \mathrm{~mm} /$ year and $\pm 1.4673 \mathrm{~mm} /$ year, respectively. In the interval $(-2,2)$ and $(-3,3)$, there are $315390(85.08 \%)$ and 351597 (97.85\%) points located, respectively. The result shows that with use of same interferogram, subsidence rates calculated from USB TCPInSAR without DEM are fairly consistent with the one calculated from conventional TCPInSAR with DEM. Without use of an external DEM to remove topographic effects, the subsidence extraction is independent of DEM errors, which means that the height errors from DEM that cannot be completely compensated by parameter estimation will not propagate into the subsidence and decrease their accuracies. Moreover, taking it into consideration that the number of TPCs are usually more than one million, it is a significant and effective way to reduce computation burden with the simplified estimation model.

\section{CONCLUSION}

This paper described an improved TCPInSAR approach with ultrashort baselines, which can detect subsidence without an external DEM and simplify the solution model. With use of 17 high resolution TSX images over northwest of TianJin China, the subsidence along A High Speed Railway between April 2009 and November 2010 were detected.

A number of TCPs are identified along the overhead bridge of A High Speed Railway, showing two subsidence funnel that would give rise to huge risk. A big subsidence funnel with subsidence of -20 -26 mm/year occurred in the ShuangJie town, 
eastern part of study area, while the relative small one with subsidence of -10 -15 mm/year occurred around the Wuqing Station. According to our investigation, Shuangjie town is experiencing the industrialization and extensive groundwater withdrawal that induced serious land subsidence. These results are validated by other studies, showing great consistencies. The detailed subsidence map was derived, which is valuable not only for government to design compensation strategies like groundwater backfill, but also to guide engineering construction,.

Moreover, the contrast between USB TCP-InSAR and conventional TCP-InSAR was made, in which the interferometric pairs are exactly the same and external DEM is only used in conventional TCP-InSAR. To make a detailed comparison 370697 common TCPs points were extracted. The mean and the standard deviation of the difference at these 370697 points are $-4.43 \times 10^{-6} \mathrm{~mm} /$ year and $\pm 1.4673 \mathrm{~mm} /$ year, respectively, indicating that even without DEM, USB TCPInSAR method can detect land subsidence accurately in flat area.

\section{Reference}

[1] Ferretti, A., Prati, C., \&Rocca, F. (2001). Permanent scatterers in SAR interferometry. IEEE Transactions on Geoscience and Remote Sensing, 39, 8-20.

[2] Berardino, P., Fornaro, G., Lanari, R., \&Sansosti, E. (2002). A new algorithm for surface deformation monitoring based on small baseline differential SAR interferograms. IEEE Transactions on Geoscience and Remote Sensing, 40, 23752383.

[3] Kampes, B. M., \& Adam, N. (2006, February). The STUN algorithm for persistent scatterer interferometry. In Fringe 2005 Workshop (Vol. 610, p. 16).

[4] Hooper, A. (2008). A multi - temporal InSAR method incorporating both persistent scatterer and small baseline approaches.Geophysical Research Letters,35(16).

[5] Mora, O., Mallorqui, J. J., \&Broquetas, A. (2003). Linear and nonlinear terrain deformation maps from a reduced set of interferometric SAR images. Geoscience and Remote Sensing, IEEE Transactions on, 41(10), 2243-2253.

[6] Hooper, A., Zebker, H., Segall, P., \&Kampes, B. (2004). A new method for measuring deformation on volcanoes and other natural terrains using InSAR persistent scatterers. Geophysical research letters,31(23).

[7] Liu, G., Luo, X., Chen, Q., Huang, D., \& Ding, X. (2008). Detecting land subsidence in Shanghai by PS-networking SAR interferometry. Sensors, 8(8), 4725-4741.

[8] CHEN, Q., DING, X. L., LIU, G. X., HU, J. C., \& YUAN, L. G. (2009). Baseline recognition and parameter estimation of persistent-scatterer network in radar interferometry.Chinese Journal of Geophysics,52(9), 2229-2236.

[9] Li, Z., Fielding, E. J., \& Cross, P. (2009). Integration of InSAR time-series analysis and water-vapor correction for mapping postseismic motion after the 2003 Bam (Iran) earthquake. Geoscience and Remote Sensing, IEEE Transactions on, 47(9), 3220-3230.

[10] Zhang, L., Lu, Z., Ding, X., Jung, H. S., Feng, G., \& Lee, C. W. (2012). Mapping ground surface deformation using temporarily coherent point SAR interferometry: Application to Los Angeles Basin. Remote Sensing of Environment, 117, 429439.

[11]Liu, G., Jia, H., Nie, Y., Li, T., Zhang, R., Yu, B., \& Li, Z. Detecting Subsidence in Coastal Areas by Ultrashort-Baseline TCPInSAR on the Time Series of High-Resolution TerraSAR-X Images.

[12] Zhang, L., Ding, X., \& Lu, Z. (2011). Modeling PSInSAR time series without phase unwrapping. Geoscience and Remote Sensing, IEEE Transactions on, 49(1), 547-556.

[13]Luo, Q., Perissin, D., Lin, H., Li, Q., \&Duering, R. (2011, June). Railway subsidence monitoring by high resolution INSAR time series analysis in Tianjin. In Geoinformatics, 2011 19th International Conference on (pp. 1-4). IEEE.

[14] Ge, D., Wang, Y., Zhang, L., Guo, X., \& Xia, Y. (2010, July). Mapping urban subsidence with TerraSAR-X data by PSI analysis. In Geoscience and Remote Sensing Symposium (IGARSS), 2010 IEEE international (pp. 3323-3326). IEEE.

[15] Lixin, Y., Fang, Z., He, X., Shijie, C., Wei, W., \&Qiang, Y. (2011). Land subsidence in Tianjin, China. Environmental Earth Sciences, 62(6), 1151-1161. 